\title{
Creatine Transporter Deficiency in Two Brothers with Autism Spectrum Disorder
}

\author{
HALIL IBRAHIM Aydin \\ From Department of Pediatrics, Medical Faculty, Section of Inborn Errors of Metabolism, Baskent University, Ankara, Turkey
}

Correspondence to:

Dr Halil Ibrahim Aydin,

Professor, Baskent University Medical

Faculty, Department of Pediatrics.

Section of Inborn Errors of Metabolism,

Temel Kuguluoglu Sokak, No: 24/2,

Bahçelievler, Ankara, Turkey.

hiaydin@yahoo.com

Received: January 12, 2017;

Initial review: May 17, 2017;

Accepted: October 25, 2017.

\begin{abstract}
Background: Creatine transporter deficiency (CTD) is a treatable, X-linked, inborn error of metabolism. Case characteristics: Two brothers with autism spectrum disorder were diagnosed with CTD at the ages of 17 and 12 years. Both were found to have a previously reported hemizygous p.408delF (c.1216_1218delTTC) deletion mutation. Outcome: Both patients were given creatine monohydrate, L-arginine, L-glycine and Sadenosylmethionine, which partially improved the behavioral problems. Message: Serum creatinine levels, creatine peak at brain MR spectroscopy or creatine/creatinine ratio in urine should be evaluated to identify CTD in children with autistic behavior and language disorders.
\end{abstract}

Keywords: Creatine deficiency syndrome, Inborn errors of metabolism, SLC6A8.
$\mathrm{C}$ reatine transporter deficiency (CTD; OMIM $300352)$ is an X-linked inborn error of metabolism caused by mutations in the creatine transporter gene (SLC6A8). SLC6A8 gene mutations impair the ability of the transporter protein to bring creatine into cells, resulting in a creatine deficiency in organs and tissues that require large amounts of energy, especially the brain [1]. Its clinical hallmarks are intellectual disability (ID), epilepsy, autistic behavior, and language disorders [2]. In this report, we describe the diagnosis and treatment of CTD in two brothers who received mild benefits from therapy despite being diagnosed late.

\section{Case Report}

The patients were two brothers, aged 17 and 12 years, from a non-consanguineous marriage with an uneventful perinatal history. Their mother did not exhibit behavioral or learning difficulties. The parents first noticed a developmental delay in the older brother at one year of age. He was diagnosed with autism spectrum disorder according to the DSM-IV criteria at the age of 2.5 years with signs of ID, severely delayed speech development, communication difficulties, swinging his trunk from side to side and a tendency to play by himself. He had four generalized tonic-clonic convulsions at seven years of age and was given valproic acid. Similarly, a developmental delay was noticed in the younger brother in his first months of life. He was diagnosed with autism at one year of age. He had a single generalized tonicclonic convulsion at 10 years of age that was not repeated after the initiation of levetiracetam. Electroencephalograms were normal for both patients.

Physical examination at admission showed no verbal expression and limited non-verbal communication with poor eye contact and inconsistent responses to instructions in both siblings. The parents noted that both siblings experienced screaming, hitting, and biting, but not selfmutilation. Other physical and clinical observations were normal without microcephaly, dysmorphy, hypotonia, myopathy, or movement disorders. They could not complete formal cognitive testing due to severe cognitive impairment.

Metabolic evaluations were normal with exception of mild pyruvic acid, 3-methylglutaconic acid, and succinic acid excretion in urine in the younger sibling. Brain MRI showed mild cerebral atrophy and mild thinning of the corpus callosum in both patients. Brain MR spectroscopy (MRS) of the patients indicated markedly decreased creatine levels in the basal ganglia and white matter. Plasma levels of creatinine were normal ( 0.50 and $0.45 \mathrm{mg} / \mathrm{dL}$, respectively), and urine creatine/creatinine ratios were increased (1.84 and 1.48 , respectively normal: $0.01-0.96)$. Guanidinoacetate levels in the urine were normal $(49.0$ and $36.0 \mathrm{mmol} / \mathrm{mol}$ creatinine, respectively; normal: 28-180). SLC6A8 gene sequencing showed a hemizygous c.1216_1218delTTC deletion in exon 8 , which resulted in the deletion of a phenylalanine (p.Phe408del). Both patients were treated with creatine monohydrate (per oral $400 \mathrm{mg} / \mathrm{kg} /$ day), Larginine and L-glycine for 14 months. S-adenosylmethionine was discontinued because of side effects. 


\section{DiscuSSION}

The reported prevalence of IEMs in autism ranges between $0.5-2.7 \%$. It is recommended to rule out metabolic disorders in autistic patients who have dysmorphism, microcephaly, ataxia, epilepsy, and severe ID, but not in patients with non-syndromic autism $[3,4]$. The metabolic investigations used for autism are serum creatinine, cholesterol, lactate, ammonia, amino acids, acylcarnitine, urine mucopolysaccharides, and organic acids [5]. The serum creatinine level should be determined in autistic children to diagnose Cerebral creatine deficiency syndromes (CDS) characterized by developmental delays, seizures, and autism. CDS can be caused by three different inborn errors of creatine synthesis and transport. The plasma creatinine level is low in patients with creatine synthesis defects, which are autosomal recessive diseases. However, this level is normal in patients with CTD. In all cases of CDS, creatine levels are low in brain tissue, as detected by MRS [6]. The serum creatinine levels of the siblings were normal, but their creatine levels in brain tissue were low. Urinary guanidinoacetate level and creatine/creatinine ratio are used for the differential diagnosis of CDS [6]. In both patients, the urine creatine/creatinine ratio was increased, and the urine guanidinoacetate level was normal, which is consistent with CTD.

The hemizygous deletion mutation (p.Phe408del) detected in the siblings has been associated with a partial or even complete loss of creatine transport function. Previously described patients with the same mutation were presented with autistic behavior pattern, severe language delay and epilepsy, as in our patients [2].

Treatment with creatine, L-arginine, and L-glycine reduced the anxiety, aggressiveness, and screaming episodes experienced by the younger sibling, but not the older sibling. We could not assess the effect of treatment on epilepsy because neither sibling had any convulsions after the initiation of antiepileptic drugs, and their electroencephalograms were normal before the treatment. Prior studies have reported that this treatment regimen can improve both behavioral and language difficulties, but we did not observe any improvements in the language skills of the patients [6]. Sadenosylmethionine was withdrawn because it increased restlessness and anxiety and reduced sleeping time when the dose was increased to $10 \mathrm{mg} / \mathrm{kg} /$ day.

Diagnosing IEMs early in life is essential for achieving prenatal diagnosis and early institution of treatment. Serum creatinine levels should be checked carefully in all patients with hypotonia, developmental delay, seizure, or autism to diagnose creatine synthesis defects, as early treatment results in excellent outcomes. In autistic patients whose serum creatinine levels are normal, if additional clinical findings such as hypotonia, developmental delay and epilepsy are present, this approach should be followed by determination of creatine peak in brain tissue by MRS in addition to other metabolic investigations.

Funding: None. Competing Interests: None stated.

\section{REFERENCES}

1. Cecil KM, Salomons GS, Ball WS Jr, Wong B, Cuck G, Verhoeven NM, et al. Irreversible brain creatine deficiency with elevated serum and urine creatine: a creatine transporter defect? Ann Neurol. 2001;49:401-4.

2. Póo-Argüelles P, Arias A, Vilaseca MA, Ribes A, Artuch $\mathrm{R}$, Sans-Fito A, et al. X-Linked creatine transporter deficiency in two patients with severe mental retardation and autism. J Inherit Metab Dis. 2006;29:220-3.

3. Ghaziuddin M, Al-Owain M. Autism spectrum disorders and inborn errors of metabolism: an update. Pediatr Neurol. 2013;49:232-6.

4. Schiff M, Benoist JF, Aïssaoui S, Boespflug-Tanguy O, Mouren MC, de Baulny HO, et al. Should metabolic diseases be systematically screened in nonsyndromic autism spectrum disorders? PLoS One. 2011;6:e21932.

5. Sudarshan S, Gupta N, Kabra M. Genetic studies in autism. Indian J Pediatr. 2016;83:1133-40.

6. Stockler-Ipsiroglu S, Van Karnebeek CD. Cerebral creatine deficiencies: a group of treatable intellectual developmental disorders. Semin Neurol. 2014;34:350-6. 